\title{
Violación del principio de proporcionalidad en la fijación de pensiones alimenticias en Ecuador
}

\section{Violation of the principle of proportionality in the establishment of alimony in Ecuador}

\author{
Marco Vinicio Cadme-Orellana \\ marco.cadme@psg.ucacue.edu.ec \\ Universidad Católica de Cuenca, Cuenca \\ Ecuador \\ https://orcid.org/0000-0002-2903-9801 \\ Cecilia Ivonne Narváez-Zurita \\ inarvaez@ucacue.edu.ec \\ Universidad Católica de Cuenca, Cuenca \\ Ecuador \\ https://orcid.org/0000-0002-7437-9880 \\ Juan Carlos Erazo-Álvarez \\ jcerazo@ucacue.edu.ec \\ Universidad Católica de Cuenca, Cuenca \\ Ecuador \\ https://orcid.org/0000-0001-6480-2270 \\ José Luis Vázquez-Calle \\ jlvazquezc@ucacue.edu.ec \\ Universidad Católica de Cuenca, Cuenca \\ https://orcid.org/0000-0003-4980-6403
}

Recibido: 13 de abril de 2020

Revisado: 01 de abril de 2020

Aprobado: 23 de mayo de 2020

Publicado: 14 de junio de 2020 


\title{
RESUMEN
}

En el presente trabajo de investigación se analizan las bases teóricas del principio de proporcionalidad, su aplicación correcta en casos particulares y la vulneración de derechos fundamentales del alimentante. Lo que se pretende es demostrar la vulneración del principio antes mencionado en menoscabo del alimentante al momento de determinar el cálculo de las pensiones alimenticias y su falta de regulación normativa. Para ello se utilizó la investigación mixta con énfasis en el enfoque cualitativo y cuantitativo, con un carácter descriptivo - explicativo, por cuanto se especificó las propiedades, características, del principio constitucional de proporcionalidad sobre pensiones alimenticias, recogidos en tratados y convenios internacionales de derechos humanos, así como en la Constitución de la República del Ecuador, con el objetivo de reformar el Código de la Niñez y Adolescencia, para que la aplicación de este principio pueda ser eficaz por parte de los administradores de justicia.

Descriptores: Derecho Constitucional; derecho a la vida; derecho a la alimentación; necesidades básicas; Desigualdad social. (Palabras tomadas del Tesauro UNESCO).

\begin{abstract}
In this research work the theoretical bases of the proportionality principle, its correct application in particular cases and the violation of fundamental rights of the feeder are analyzed. What is intended is to demonstrate the violation of the aforementioned principle to the detriment of the food provider when determining the calculation of alimony and its lack of regulatory regulation. For this, mixed research was used with an emphasis on the qualitative and quantitative approach, with a descriptive - explanatory nature, since the properties, characteristics, of the constitutional principle of proportionality on alimony, specified in international human rights treaties and conventions, were specified, as well as in the Constitution of the Republic of Ecuador, with the objective of reforming the Childhood and Adolescence Code, so that the application of this principle can be effective by the administrators of justice.
\end{abstract}

Descriptors: Constitutional right; right to life; right to food; basic needs; social inequality. (Words taken from UNESCO Thesaurus).

\section{INTRODUCCIÓN}

El principio constitucional de proporcionalidad, entendido como un principio de carácter general que tiene por objeto crear límites a las actuaciones de los encargados de administrar justicia con el fin de evitar que se vulneren derechos fundamentales, está conformado por criterios de idoneidad, necesidad y proporcionalidad en sentido estricto. El derecho de alimentos se relaciona tanto con el derecho a la vida, la supervivencia y a 
una vida digna. En este contexto, se debe tener en cuenta que el cálculo de las pensiones alimenticias en Ecuador se lo hace a través de una tabla con valores mínimos y máximos; y de acuerdo con 6 tipos de niveles que se establecen según la remuneración del alimentante, edad y número de hijos.

En un proceso judicial la obligación que tiene el progenitor de pasar alimentos debe cumplir con la proporcionalidad debida, es decir que la cantidad que se fija de pensión alimenticia sea acorde a la capacidad económica del alimentante para cumplir con esta obligación. El principio del interés superior del niño siempre va a proteger los intereses de los menores por ser considerados dentro del grupo de atención prioritaria, sin embargo, no es un principio absoluto, sino más bien cede frente a la propia subsistencia del alimentante como lo señala la sentencia emitida por la Corte Constitucional (Corte Constitucional, 2012), es imperativo relativizar este principio de manera que la ley contemple circunstancias extraordinarias como el tema de la capacidad económica del alimentante, ya que al no tener lo necesario para subsistir no podría cumplir con el pago de las pensiones alimenticias.

La sentencia emitida por la Corte Constitucional No.048-13-SCN-CC resolvió en su segundo inciso lo siguiente: para que se determine el ingreso del alimentante para la aplicación del porcentaje que corresponda se efectuará deduciendo previamente el aporte del trabajador a la seguridad social (Corte Constitucional, 2013).

El problema surge ya que en la actualidad, para hacer el cálculo de las pensiones alimenticias se toman en cuenta únicamente los ingresos del alimentante, menos el aporte al IESS y el impuesto a la renta, sin considerar otras circunstancias adicionales, como deudas crediticias e hipotecarias contraídas para el beneficio del menor beneficiario; otro problema que se evidencia es el tema de las pensiones alimenticias adicionales, cuando en ocasiones el alimentante vive del trabajo diario y no alcanza a cubrir el monto de la pensión alimenticia que ha sido fijado por el juez.

Por los antecedentes expuestos, se planea como problema de investigación: ¿Cómo afecta la vulneración del principio de proporcionalidad en la fijación de la tabla de pensiones alimenticias sin considerar los gastos que van a beneficio del alimentado que 
Marco Vinicio Cadme-Orellana; Cecilia Ivonne Narváez-Zurita; Juan Carlos Erazo-Álvarez; José Luis Vázquez-Calle

no son deducibles por la norma? por consiguiente, el objetivo de la investigación consiste en: proponer la reforma a la determinación de pensiones alimenticias establecidas en el artículo enumerado 15 y 16 correspondiente al Título V del Libro II del Código Orgánico de la Niñez y Adolescencia como garantía del principio de proporcionalidad del alimentante.

\section{Referencial teórico}

\section{La jurisprudencia constitucional y el principio de proporcionalidad}

Los autores (Estudios Constitucionales, 2012); (Cárdenas, 2014); y, (López Idalgo, 2017) mencionan que el principio de proporcionalidad se origina en el derecho prusiano de policía donde la proporcionalidad se orientaba al respeto de las libertades individuales. La jurisprudencia del Tribunal de Prusia sostuvo que este concepto en principio ligaba sólo al poder ejecutivo y controlaba el ejercicio del poder de policía. En consecuencia, el principio de proporcionalidad se da en una época en que el Derecho Constitucional no respondía a esa realidad, ya que el legislador no se sujetaba a la Constitución, en este sentido, sólo al ejecutivo se le aplicaba este criterio de proporcionalidad; como resultado, los derechos fundamentales no gozaban de valor normativo ni de eficacia jurídica; más bien primaba un criterio de legalidad que el de constitucionalidad y en un Estado de Derecho la importancia de la supremacía constitucional es primordial.

A título ilustrativo, se precisa que fue en Europa y después de la segunda guerra mundial que cobra fuerza el principio de proporcionalidad, para desarrollarse como un método de interpretación aplicado por jueces de las distintas ramas del Derecho, Doctrina y Jurisprudencia; incorporándose en las constituciones modernas, para evolucionar y adaptarse a la realidad. En cuanto a su ámbito de aplicación países europeos como Alemania son los precursores, para luego llegar al sistema interamericano de derechos humanos y en lo posterior a América; el principio de proporcionalidad se caracterizaba por el hecho de que se reconocían derechos para su protección jurídica.

Lo sustancial del principio de proporcionalidad es el control que se hace a las actuaciones excesivas por el poder público, de manera que el criterio proporcionalidad se extiende y 
evoluciona en la medida que pasa a convertirse en un principio constitucional de protección de los derechos fundamentales (Estudios Constitucionales, 2012). El significado de proporcionalidad engloba aquello que guarda equivalencia, correspondencia, equilibrio. La proporcionalidad indica un vínculo de equilibrio entre dos objetos (Becerra, 2019). A este respecto, lo verificable a lo proporcional es equiparable a lo que se entiende como equivalencia que existe entre sí, no sólo tiene que ver con objetos, pues la proporcionalidad tiene un sentido más amplio que engloba un sinnúmero de posibilidades y puede ser utilizado en varias materias.

En sentido amplio, según (Barnes, 1994) por proporcionalidad se entiende la prohibición en exceso cuando el objetivo es alcanzar un fin necesario y la única manera de hacerlo es restringir otra medida que sea menos perjudicial, para satisfacer en mayor grado la garantía de los derechos fundamentales, como la libertad de las personas; y en ese mismo contexto, la proporcionalidad en sentido estricto pondera entre dos derechos para escoger el que más beneficie el interés general.

Lo esencial de este principio es la libertad de los ciudadanos y el pleno goce de los derechos fundamentales desarrollados por la Constitución, restringiendo un derecho por otro que brinde mayor eficacia, es decir que prime el interés general sobre el particular. Es necesario, tomar en cuenta que la acción estatal en cualquiera de sus formas de expresión posible, sea acto administrativo o resolución judicial debe ser útil, necesaria y proporcionada; esto como un aspecto importante para el objetivo en estudio.

El principio de proporcionalidad de acuerdo con el criterio de (Benalcazar, 2018) es una técnica interpretativa que tiene el Derecho Constitucional, con el fin garantizar los derechos fundamentales de los ciudadanos al momento de ser aplicado, así mismo, tiene la función de controlar los actos del poder público para impedir que los derechos antes mencionados no sean vulnerados. La proporcionalidad debe ser interpretada por la autoridad jurisdiccional, por lo que es importante la motivación por cuanto puede dar fuerza al argumento aplicado a un caso concreto. El principio de proporcionalidad según (Vilela, 2014) se caracteriza por tener tres requisitos básicos: 
Marco Vinicio Cadme-Orellana; Cecilia Ivonne Narváez-Zurita; Juan Carlos Erazo-Álvarez; José Luis Vázquez-Calle

a) Idoneidad.- Todas las medidas que no sean necesarias para conseguir la consecución de un fin serán rechazadas. Siempre primará el requisito de idoneidad cuando existan dudas entre principios, sin ser necesario que se cumpla una finalidad total, sino más bien lo imperativo será el cumplimiento de fines parciales.

b) Necesidad.- Esta regla se da cuando la medida de control tomada, no es la más eficaz en aras de la consecución de un fin, en este caso se debe aplicar la medida que pueda afectar en menor grado que va a ser la más beneficiosa para la persona.

c) Proporcionalidad en sentido estricto.- Este requisito no se desarrolla en el ámbito de lo real sino más bien en un plano netamente jurídico. La pretensión consiste en acomodar el derecho que exige protección con otros derechos y con el bien común.

El alcance de lo idóneo es amplio, no solo hay que invocarlo, sino debe estar motivado en forma correcta, de manera que reúna las condiciones necesarias u óptimas para una función o fines determinados; y para eso no basta que se cumpla con un fin completo, sino es suficiente que se cumplan con un fin parcial. La necesidad rechaza una medida tomada; y la cambia por un modo alternativo que en sí puede perjudicar en menor medida de lo posible a la persona; para que en el último requisito se centre de manera primordial en el plano de lo jurídico y se armonice el derecho que es objeto de protección frente a otros derechos y el bien común.

La importancia del principio de proporcionalidad es la de "limitar la injerencia del Estado en la afectación de los derechos fundamentales de los ciudadanos, se debe aplicar la proporcionalidad para evaluar la constitucionalidad de una medida restrictiva de derechos fundamentales" (Cáceres, 2016, p.20). Este principio es un método que no siempre llegará a ser certero y pleno en cuanto a satisfacer a totalidad un objetivo, siempre tratará de brindar dignamente una racionalidad cuando exista confrontaciones entre reglas 0 principios constitucionales, no se aspira a encontrar una sola respuesta que sea la ideal, sino más bien tiende a buscar una decisión que sea la correcta, o por lo menos unas que 
se justifiquen (Cárdenas, 2014).

La relación que existe entre el principio de proporcionalidad con la ponderación es importante, ya que, los principios al ser supuestos de hecho, no tienen una clara consecuencia, es decir, pueden ser aplicadas e interpretadas de varias formas, por lo que es primordial utilizar la ponderación para resolver este problema (Cáceres, 2016). Cuando se utiliza la ponderación se enfrenta por un lado a dos normas tipo principio, sin embargo, la idea no consiste en someter a una sobre la otra, pues esa forma de aplicación ocurre con las reglas, su finalidad radica en restringir una en favor de otra, de manera que se le dota de más validez en un caso concreto.

La ponderación debe ser entendida como un método encaminado para la aplicación de principios que en su estructura contiene mandatos de optimización y consiste en la medida de decisión que tiene el juez cuando existe contradicción de derechos y principios que concurren en el caso específico. El conflicto de principios y su ponderación correspondiente se desarrolla en dos momentos: primero cuando el legislador tiene la necesidad de sacrificar en mayor o menor medida un principio en desarrollo de otro, y el segundo que se da al momento que el juez va a dictar su decisión. En un caso específico al existir un conflicto entre dos principios constitucionales se aplicará uno de los dos en sacrificio del otro, por cuanto no pueden aplicarse al mismo tiempo por existir contradicción de principios (Tamayo, 2011).

En la Constitución de la República del Ecuador el principio de proporcionalidad está contemplado en el Art. 76 en los procesos que establezcan deberes y derechos de cualquier índole, garantizando el debido proceso que además, consta de garantías esenciales. El numeral 6 de la presente norma determina la proporcionalidad debida que existe entre las sanciones e infracciones penales, o de cualquier materia e incluso en el ámbito administrativo (Asamblea Nacional Constituyente, 2008).

El texto normativo constitucional determina la proporcionalidad debida que existe como garantía del debido proceso, y además el Estado ecuatoriano tomará en cuenta los límites que conlleva este principio para ser aplicado a un caso en concreto, motivando sus decisiones para que los derechos fundamentales no sean violentados de una forma 
arbitraria. En este mismo contexto, el Art. 8 de la Declaración Universal de Derechos Humanos establece que las personas pueden acceder e interponer recursos ante las judicaturas que tengan la competencia debida, de manera que se garanticen los derechos reconocidos por la carta magna cuando se hayan violado derechos fundamentales por cualquier acto ilegítimo que les cause perjuicio (Asamblea General de las Naciones Unidas, 1948).

En convenios internacionales, es necesario que se contemple y garantice el derecho que tienen las personas para acudir a los órganos competentes del Estado, para que se respeten los principios contemplados en cada ordenamiento jurídico interno en amparo de la protección necesaria de los derechos fundamentales de las personas. Por otro lado, la Ley Orgánica de Garantías Jurisdiccionales y Control Constitucional (LOGJCC) en su Art. 3 establece que todas las normas contenidas en la Constitución se deben interpretar en la forma que mejor se ajuste a la carta magna en su integralidad, en efecto, cuando exista duda, se interpretará en un sentido que más beneficie los derechos que se reconocen en la Constitución y respete la voluntad del pueblo.

Dentro de este marco, el segundo inciso de la ley en mención indica como método de interpretación el principio de proporcionalidad y; establece que dé existir un conflicto surgido entre normas o principios que no se pueda resolver a través de las reglas de antinomias, se aplicará este principio (Asamblea Nacional del Ecuador, 2009). El principio de proporcionalidad se debe aplicar cuando exista contradicción entre normas, sin embargo, se debe tener en cuenta la importancia del mismo, puesto que el fin que persigue no es más que garantizar los derechos fundamentales.

\section{El interés superior del niño en relación al principio de proporcionalidad. Regulación constitucional y enfoque internacional}

La Constitución de la República del Ecuador en su Artículo 44 determina que "(...), se atenderá al principio de su interés superior y sus derechos prevalecerán sobre los de las demás personas" (Asamblea Nacional Constituyente, 2008, art 44). El Estado garantiza el interés superior del niño y expresa, por otra parte, que estos derechos están por sobre 
las demás personas, como una forma de protección especial del ente estatal por ser un grupo de atención prioritaria, así mismo, el Estado garantiza el desarrollo integral de los niños, niñas y adolescentes.

El desarrollo integral debe ser entendido como el desenvolvimiento del menor tanto en el plano físico como emocional, ese proceso va desde el crecimiento, maduración, hasta las aspiraciones que pueda llegar a tener en un futuro, todo esto dentro de un ambiente familiar y social, garantizando un marco de seguridad y protección del menor. La norma Constitucional expuesta, se consolida como una estrategia garantista a favor de los menores, "garantismo designa una filosofía política que impone al derecho y al Estado la carga de la justificación extrema conforme a los bienes y a los intereses cuya tutela y garantía constituye precisamente la finalidad de ambos" (Ferrajoli, 2004, p.853).

En respuesta a las consideraciones expuestas, los jueces al momento de decidir en un caso concreto, fundamentarán este principio en sus decisiones, es decir, justificando el motivo que conllevó a tomar ese fallo, por supuesto, basándose siempre en criterios jurisprudenciales y normativos. Por otro lado, el ámbito de aplicación de los jueces es muy amplio, y se da de manera discrecional.

En la medida que corresponde a la autoridad jurisdiccional (Juez) tomar decisiones por los menores, lo que se traduce a la subjetividad, teniendo en cuenta que su interpretación es extensa y para hacerlo, debe construirse de manera que justifique la decisión jurisdiccional en un caso concreto (Almeida, Erazo, Ormaza, \& Narváez, 2019), y no se torne en un abuso excesivo por parte del poder judicial. Su aplicación puede originar una restricción de derechos, de tal forma que, pueden aprovecharse y limitarse otros intereses que estén en pugna (Simón, 2013). Existe un temor fundado a excusa del principio de interés del niño como derecho absoluto, y la vulneración de otros derechos como en este caso, el del alimentante; que este interés sea usado para "justificar preferencias, prejuicios, opciones personales, aprovechándose de la indeterminación del concepto y las facultades discrecionales que se otorgan al juez para decidir su alcance al caso concreto" (Simón, 2013, p.151). En el contexto latinoamericano y de manera particular en el Ecuador no se puede aceptar amenazas venidas de intereses individuales producto de 
Marco Vinicio Cadme-Orellana; Cecilia Ivonne Narváez-Zurita; Juan Carlos Erazo-Álvarez; José Luis Vázquez-Calle

la arbitrariedad en la aplicación de los derechos por el poder judicial.

Bajo estas consideraciones, ¿es posible concebir la existencia de límites en su aplicación?, claro que es posible, pues si bien, el interés superior del niño defiende un interés privado "el amparo de un interés no puede desconocer la existencia de otros derechos que los adultos son titulares y de la misma manera merece la debida protección" (Vilela, 2014, p.161). El interés superior del niño es primordial, sin embargo, no por ese hecho, se puede desconocer una realidad que es palpable, puesto que, no es un principio absoluto y no se puede alegar este criterio y desconocer el derecho que tiene el alimentante a vivir dignamente, es decir, con lo necesario para su propia subsistencia, además, a los menores los cobija una gama amplia de derechos, de manera que nunca se los deja en la indefensión.

Se debe entender que la Constitución obliga a los jueces el deber de motivación de las resoluciones y no bastará simplemente con hacer una declaración de la superioridad de este principio, sino se debe justificar su aplicación, tal como señala Igartúa (1998) "contra el riesgo de la arbitrariedad no se conoce otro antídoto que la motivación" (p.77). De lo que se mencionó, se observa que el interés superior del niño constituye un derecho superior en las decisiones de múltiples materias, sean esas administrativas, legislativas y judiciales, además al momento de alegar la debida proporcionalidad entre un hecho y un derecho, se tomará en cuenta los requisitos indispensables que requiere este principio, de manera que pueda ser eficaz en cumplimiento de los derechos fundamentales, la idoneidad, necesidad y proporcionalidad en sentido estricto.

El derecho de alimentos es una obligación solidaria y por lo mismo, no solo debe asumirla el padre, sino la madre, familiares, el Estado y las instituciones públicas y privadas; al respecto, se puede evidenciar que para el cumplimiento no sólo al derecho de alimentos, sino al "cúmulo de derechos que le asisten a los menores, el Estado ecuatoriano a través de instituciones públicas, así mismo personas jurídicas privadas han tutelado los derechos del menor, pudiendo acceder a programas de salud provenientes del Estado ecuatoriano" (Corte Constitucional, 2012, p.32). 
Atendiendo a estas consideraciones, es responsabilidad de los padres velar por el mantenimiento de los hijos. La subsistencia de los niños, niñas y adolescentes no solo está consagrado en la Constitución del Ecuador, sino en instrumentos internacionales como la Convención sobre los Derechos del Niño, la misma que hace referencia al principio del interés superior del niño que en su artículo 3 que dispone: "En todas las medidas concernientes a los niños que tomen las instituciones públicas o privadas de bienestar social, los tribunales, las autoridades administrativas o los órganos legislativos, una consideración primordial a que se atenderá será el interés superior del niño" (Organización de las Naciones Unidas, 1989, art 3).

El interés superior del niño no solo aparece en la Convención, sino desde la misma declaración de los derechos del niño, con un carácter consuetudinario antes que normativo, a través de los cuales, varios países de Latinoamérica cambiaron su normativa respecto a la protección de los menores, como también lo hizo el Estado ecuatoriano en su denominado nuevo código de menores. El Ecuador consagra este principio del interés superior del niño en la Constitución de la República, sin embargo, este principio no nace de las necesidades de la colectividad o porque la realidad social así lo requería, sino porque el Ecuador pasó a suscribir tratados internacionales y ser parte de ellos; y esto obligo a adecuar su normativa interna con los tratados internacionales para tomar mayor interés por parte de los legisladores y crear políticas a favor a este grupo de atención prioritaria.

Partiendo de los supuestos anteriores, la convención precisa en su artículo 28 inciso 4, la obligación que tienen los Estados parte que lo integran, para que tomen todas las medidas adecuadas, y así, certificar que se cumpla con el pago de las pensiones alimenticias, sea por parte de los obligados principales o subsidiarios de ser el caso, sin importar que vivan dentro del territorio nacional o se halle radicados en el exterior (Organización de las Naciones Unidas, 1989). De lo estudiado por la jurisprudencia contenida en tratados y convenión internacionales, lo más importante radica en que al menor no se lo deje en indefensión. La norma no debe tomar en cuenta solo las facultades económicas del alimentante, sino las necesidades del alimentario para subsistir y que 
Marco Vinicio Cadme-Orellana; Cecilia Ivonne Narváez-Zurita; Juan Carlos Erazo-Álvarez; José Luis Vázquez-Calle

correspondan a su posición social de manera que se garantice una debida proporcionalidad.

\section{El Derecho de alimentos y su fijación en base al principio constitucional de proporcionalidad. Análisis y crítica a la tabla de pensiones alimenticias}

El derecho de alimentos que gozan los menores, es esencial y viene intrínseco a la persona, tiene diferentes características que lo diferencian de los demás derechos como el ser imprescriptible, no se puede renunciar, es inembargable, y su violación limita la supervivencia de los menores, sean estos niños, niñas y adolescentes. Sus derechos se encuentran garantizados en el Código de la Niñez y Adolescencia, la Constitución y en tratados y convenios internacionales.

El proveer alimentos no sólo consta de una obligación que tiene que asumir el padre, pues la madre también se encuentra obligada a prestar los alimentos y en ausencia de ellos los obligados subsidiarios conforme dispone la ley. La finalidad del derecho de alimentos es solventar las necesidades básicas del alimentario sobre la base de su desarrollo integral. Se necesita dar a conocer que toda persona que presta alimentos tiene derechos y debe vivir dignamente, y por lo mismo, no se debe vulnerar un principio fundamental contemplado en la Constitución, como el principio de proporcionalidad.

En consecuencia, el cálculo las pensiones alimenticias se determinan a través de una tabla con valores mínimos y máximos, de acuerdo con 6 tipos de niveles que se establecen según lo que perciba de remuneración el alimentante, otros factores como las edades y número de hijos e hijas a quienes deba prestar los alimentos el progenitor (Ministerio de Inclusión Económica y Social, 2020). Es importante hacer una investigación real de los ingresos a percibir por parte del alimentante, para determinar la pensión definitiva que se le va a imponer en un caso concreto, por lo que esa problemática se relaciona con lo que dispone la sentencia 048-13-SCN-CC, en su segundo considerando establece que: se deducirá el aporte que se realice del trabajador al IESS, esto para determinar la aplicación de la tabla de pensiones (Corte Constitucional, 2013). 
Es evidente que la Corte Constitucional solo toma en cuenta para este aspecto, la deducción del aporte al IESS, no hace referencia a otras circunstancias que se pueden presentar como contraer deudas para el beneficio de la familia del beneficiario, es decir, del alimentado; ya que esta situación no se encuentra contemplada por la ley.

Por otra parte, el Consejo Nacional de la Niñez y Adolescencia (CNNA) en su resolución 001-CNNA-2013 expide la tabla de pensiones alimenticias, señala los parámetros que deben cumplirse para su elaboración, los mismos que están distribuidos de la siguiente manera: las necesidades esenciales primordiales del alimentado, los ingresos ordinarios como extraordinarios del alimentante, gastos propios, de sus dependientes directos, la distribución del consumo familiar, ingresos de los alimentantes, derechohabientes, por último la inflación (Consejo Nacional de la Niñez y Adolescencia, 2013).

Es trascendental considerar la primacía del interés superior de los menores, para garantizar la realización de los fines que persigue esta obligación, como ya se ha explicado en líneas anteriores, en ese intento por no dejar en la indefensión a los menores, se ignoró el derecho a vivir dignamente que tiene el padre o madre que presta los alimentos y se ha criticado duramente esta falta de reglamentación tanto por jueces como abogados en libre ejercicio.

El problema se genera cuando se adquiere deudas crediticias; en un caso concreto, si se toma en cuenta que una persona gana 800 usd, es decir, dos salarios básicos unificados, se colocaría en el nivel uno de la tabla, y si tuviese dos hijos mayores de 5 años el aporte seria del $43.13 \%$ del ingreso que percibe, casi la mitad del sueldo del alimentante, y si a eso se adiciona que su casa está hipotecada y paga 300usd mensuales por 20 años, prácticamente el concepto de una vida digna se acaba y se ve reflejada una completa desproporcionalidad, pues la tabla no toma en cuenta los descuentos por conceptos de deudas, y el juzgador se ve obligado a aplicar la misma por falta de disposición escrita. El interés superior del niño prima por encima de los demás derechos como regla general, sin embargo, esta investigación viste de tal importancia, puesto que, en ocasiones no cuenta el alimentante con los recursos necesarios para prestar alimentos, e incluso se encuentra desempleado y al momento de fijar los valores de la pensión alimenticia no 
existe la debida proporcionalidad con los ingresos reales del alimentante.

En relación con la problemática expuesta, (Auris, 2018) en su investigación analiza el pago de las pensiones alimenticias adicionales referentes al décimo tercer y décimo cuarto, aborda además, el problema del alimentante desempleado que conlleva a un alto índice de retraso en el pago de las pensiones alimenticias adicionales, vulnerando así el principio de proporcionalidad que contempla el artículo 76 numeral 6 de la Constitución de la República.

En este contexto, el artículo innumerado 16 inciso 2 Título $V$ del Código de la Niñez y Adolescencia, manifiesta que, el alimentado recibirá el pago de dos pensiones adicionales que se cancelarán en el mes de septiembre - diciembre de cada año en lo que tiene que ver al régimen de la Sierra, así mismo en los meses de abril y diciembre la Costa y Galápagos deberán cancelar el pago de estos montos en los términos establecidos en esta ley, y si el alimentante no labora en relación de dependencia, esto no le exonera del pago al que se ve obligado (Congreso Nacional, 2003).

Al respecto, (Zaidán \& Simón, 2020) manifiestan que mediante acuerdo ministerial se puede adecuar la tabla de pensiones alimenticias y adaptarla a la realidad ecuatoriana. La vulneración al principio de proporcionalidad no sólo pasa porque se determine como deducción únicamente el aporte al seguro social, también por el hecho de los décimos, específicamente la décima tercera y cuarta remuneración, en este caso el alimentante debe duplicar esa pensión, sin importar cuanto es lo que percibe mensualmente y tomando en cuenta que no todos trabajan en relación de dependencia, lo cual le pone en una situación de vulnerabilidad de derechos fundamentales.

Finalmente, cabe resaltar que, la concreción del principio de proporcionalidad, en ocasiones invocado por la Corte Constitucional del Ecuador, ha sido confusa y ha servido como enunciado en varias de sus sentencias, debido a que su discrecionalidad es extensa. Es pertinente mejorar de manera técnica el uso de este principio, a fin de que dote más racionalidad a los fallos que puedan adoptar de aquí en adelante la Corte Constitucional para garantizar su legitimización efectiva (Mogrovejo, Erazo, Pozo, \& Narváez, 2019). Todo esto dentro de un marco de justicia social y protección de derechos, 
en este sentido, los jueces aseguren que el acceso a la justicia sea lo más correcto posible, que la aplicación de este principio se base en la igualdad, integridad y protección de los derechos fundamentales tanto de los obligados a prestar alimentos, como los beneficiarios de este derecho.

\section{MÉTODO}

El presente estudio transversal presentó un carácter descriptivo - explicativo, por cuanto se especificó las propiedades y características del principio constitucional de proporcionalidad y de pensiones alimenticias, recogidos en tratados y convenios internacionales de derechos humanos, así como en la Constitución de la República del Ecuador. Además, logró un alcance explicativo por cuanto se estableció la relación de las variables, determinando las causas del fenómeno en estudio (vulneración del principio de proporcionalidad). La información fue levantada mediante la revisión, análisis, de tratados y convenios internacionales, Constitución de la República del Ecuador, Código de la Niñez y Adolescencia, libros y artículos publicados en revistas científicas indexadas en Dialnet, luris Dictio, revistas jurídicas, entre otros.

El tipo de investigación fue mixto, con énfasis en el enfoque cualitativo, ya que en la primera fase se procedió con la revisión de conceptos, evolución histórica, formas de garantía y mecanismos que pueden incidir en la disminución del problema en estudio, mientras que, en la segunda fase se utilizó herramientas cuantitativas como la encuesta para el levantamiento de datos (Hernández, Fernández, \& Baptista, 2014).

Los métodos aplicados en esta investigación fueron: histórico - lógico sustentado a través del tiempo, de manera cronológica, dentro de un orden jurídico y vigente, apoyado por la jurisprudencia y doctrina (Villabella, 2015); el analítico - sintético facultó el análisis de fuentes primarias y secundarias, para dotarlas de relevancia jurídica, así defender el objetivo planteado, sostener las ideas más importantes que motivaron la investigación, considerando el ordenamiento jurídico ecuatoriano (Behar, 2008); el método inductivodeductivo, permitió el razonamiento para establecer generalizaciones respecto a la problemática del principio de proporcionalidad y su vulneración al momento de la 
aplicación a la tabla de pensiones alimenticias.

El universo de estudio de la investigación estuvo conformado por 9 jueces de la unidad de familia y 7 abogados en libre ejercicio de su profesión, seleccionados a través de un muestreo por conveniencia considerando los criterios de facilidad de acceso, disponibilidad de personas e intervalo de tiempo.

\section{RESULTADOS}

Una vez obtenida la información a través de las encuestas realizadas sobre el tema de la violación del principio de proporcionalidad en la fijación de pensiones alimenticias en el Ecuador, es necesario dotar a los resultados de un enfoque dentro del marco del Derecho Constitucional, de manera que fortalezca el ámbito de aplicación de este principio, para evitar la vulneración de derechos fundamentales del alimentante al momento de la aplicación de la tabla de pensiones alimenticias.

El estudio fue realizado con fines académicos, en este sentido, la encuesta consideró la intervención de jueces de notable reconocimiento, así como la participación de abogados en libre ejercicio de su profesión, su diseño se estructuró con 21 preguntas cerradas y sus alternativas posibles de respuesta. Entre los principales resultados se determinó:

\section{Conocimiento de los jueces sobre el principio de proporcionalidad}

Un alto grado de conocimiento por parte de los jueces (89\%) sobre el principio de proporcionalidad.

\section{Cálculo de las pensiones alimenticias}

En lo referente al cálculo en la tabla de pensiones alimenticias y vulneración al principio constitucional de proporcionalidad del alimentante, el 55,6\% de jueces encuestados coincidió en que si existe vulneración, mientras que, el 44,4\% señaló que no (ver figura 1), estos resultados dejan en evidencia los criterios divididos con relación a la existencia de la vulneración de este principio, en este sentido, resulta trascendental el rol de los jueces al momento de decidir en un caso concreto, ya que la deficiencia de la 
proporcionalidad en la práctica genera subjetividad, y por lo mismo carencia de legitimidad del principio.

Por su parte, los abogados de libre ejercicio en su mayoría representados por un $71,4 \%$, concuerdan en que casi siempre los jueces vulneran el derecho constitucional de proporcionalidad del alimentante al fijar el derecho de alimentos, en tanto que, el $14,3 \%$ de profesionales aseguran que no existe vulneración del derecho en análisis.

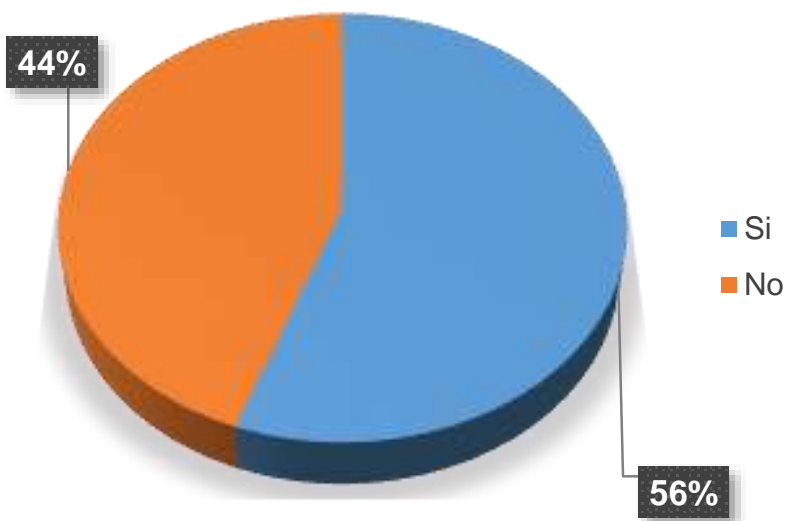

Figura 1. Vulneración del principio constitucional de proporcionalidad. Fuente. Encuestas realizadas a los jueces y abogados en libre ejercicio.

\section{Pensiones alimenticias adicionales}

Una implicación más de los criterios divididos de los jueces que tienen sobre la determinación de las pensiones alimenticias se refleja en el pago de pensiones adicionales, pues sólo el 44,4\% consideran que las pensiones adicionales (décimo tercera y cuarta remuneración) vulneran el principio constitucional de proporcionalidad. Esto significa por una parte que el criterio de la mayoría de los jueces determina justo el pago de estos valores adicionales, y por otra parte, se evidencia la necesidad de adecuar una normativa que permita aplicar de forma eficaz el principio de proporcionalidad que consagra la Constitución de la República del Ecuador. 


\section{Interés superior del niño}

Sobre la determinación de si el interés superior del niño es absoluto, el $78 \%$ de profesionales manifestaron que no lo es (ver figura 2), eso explica que a este principio se le pueden establecer límites.

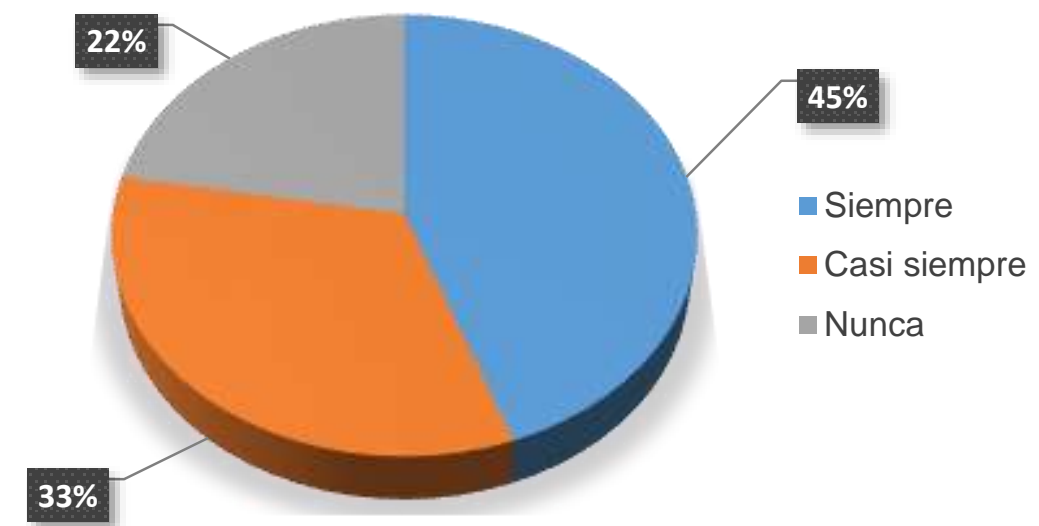

Figura 2. Limites en aplicación del interés superior del menor. Fuente. Encuestas realizadas a los jueces y abogados en libre ejercicio.

\section{Deducción previa del aporte del trabajador a la seguridad social}

Otro aspecto importante se refiere al cálculo de las pensiones alimenticias, ya que para determinar el ingreso se basa sólo en la deducción previa del aporte del trabajador a la seguridad social y no se toma en consideración los créditos adquiridos y empleados en beneficio de los alimentantes, a este respecto, las dos unidades de análisis coinciden de forma mayoritaria; por un lado, el $66,7 \%$ jueces, y por otro, el $86 \%$ de abogados están de acuerdo en que no basta como único criterio la deducción del aporte al IESS, este resultado marca un hito, en el sentido de que la norma vigente no es eficaz, sin embargo, al no estar regulado este particular los jueces deben aplicar la norma aun cuando es evidente la vulneración del principio de proporcionalidad del alimentante. 


\section{Alternativas o medios a considerar para evitar la vulneración del principio}

Los medios para resolver la problemática a la vulneración del principio se sustentan en diferentes posibilidades, de aquí que los jueces optaron de forma indistinta en la selección de las alternativas presentadas, no obstante, el $56 \%$ de ellos (ver figura 3 ) concuerdan en que la solución se debe proponer a través de una reforma legislativa en sus innumerados 15 y 16 del Libro II del Código Orgánico de la Niñez y Adolescencia; con este resultado no se descartan las demás opciones, empero, se reafirma la vulneración permanente al principio de proporcionalidad en la determinación de las pensiones alimenticias.

d) Ninguna de las anteriores

c) Mediante acuerdo ministerial que reforme la tabla de pensiones alimenticias

b) Mediante sentencia emitida por Corte Constitucional que establezca circunstancias ...

a) Mediante reforma legislativa en sus innumerados 15 y 16 de la Ley Reformatoria al Título IV del Libro.

\section{$11 \%$}

\section{$22 \%$}

\section{$11 \%$}

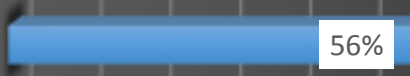

$\begin{array}{lllllllllll}0 & 0,5 & 1 & 1,5 & 2 & 2,5 & 3 & 3,5 & 4 & 4,5 & 5\end{array}$

Figura 3. Alternativas de solución al problema de la vulneración. Fuente. Encuestas realizadas a los jueces y abogados en libre ejercicio.

De los resultados obtenidos se deduce que el conocimiento de jueces y abogados profesionales sobre el principio de proporcionalidad es alto, sin embargo, existe discrepancia entre los participantes de las dos unidades de análisis establecidas. Esta apreciación conduce a la investigación a un escenario que tiene que ver con el debate actual, de manera particular con la situación laboral de los alimentantes, específicamente de quienes no tienen salario fijo por no trabajar en relación de dependencia o de quienes mantienen créditos bancarios por la adquisición de bienes a favor y disfrute de sus alimentados, pues no considerar estas situaciones conlleva a una afectación económica y social del alimentante así como a la vulneración del principio constitucional en estudio. Por tanto, se persigue mediante esta investigación una propuesta jurídica que proteja al 
niño sin vulnerar sus derechos y a la par permita el mejoramiento de las condiciones de vida del alimentante y su familia mediante la fijación adecuada de la pensión alimenticia en términos de eficacia y proporcionalidad.

\section{PROPUESTA}

De acuerdo con los resultados obtenidos, la problemática es clara y consta de la vulneración del principio de proporcionalidad del alimentante en la aplicación de la tabla de pensiones alimenticias, conforme se ha ido desarrollando el trabajo de investigación la siguiente propuesta se hace sobre la base de una reformatoria de la Ley al Código Orgánico de la Niñez y Adolescencia conforme a los parámetros expuestos en la figura 4:

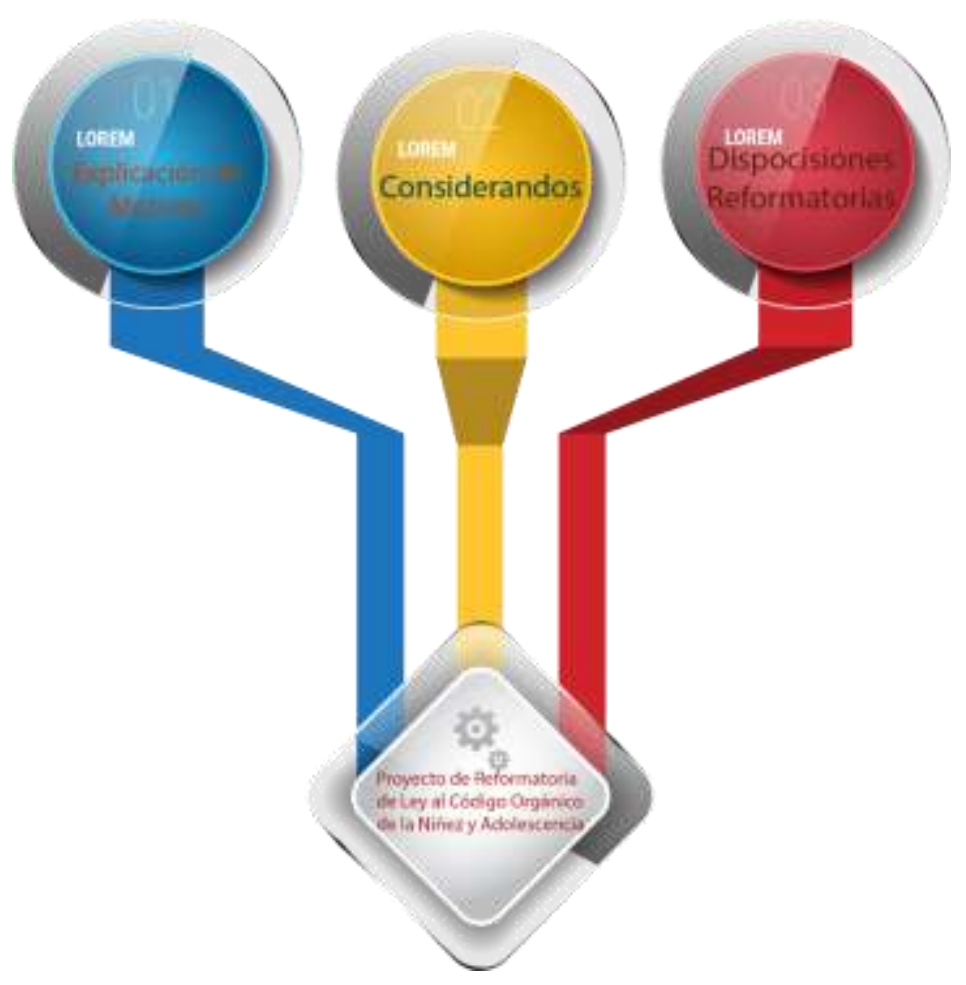

Figura 4: Esquema de propuesta de Ley Reformatoria al Código Orgánico de la Niñez y Adolescencia 


\section{Proyecto de Ley Reformatoria al Código Orgánico de la Niñez y Adolescencia Exposición de motivos}

Al haberse demostrado la vulneración del principio de proporcionalidad en la aplicación de la tabla de pensiones alimenticias por no tomarse en cuenta la capacidad económica del alimentante, se hace necesario garantizar derechos tanto para alimentante cuando para alimentado, lo que tiene como objetivo central la exigencia del requisito de razonabilidad que deben gozar los actos y relacionados con la idea de justicia, para asegurar de que las decisiones de los jueces se basen en principios que tengan como objetivo el cumplimiento de fines conforme a la Constitución.

En ese sentido, se requiere señalar que el artículo 15 del Código Orgánico de la Niñez y Adolescencia y la sentencia de la Corte Constitucional No.048-13-SCN-CC mencionan que la determinación del ingreso del alimentante para la aplicación del porcentaje que corresponda se efectuará deduciendo previo el aporte del trabajador a la seguridad social (Corte Constitucional, 2013), situación que demuestra que no se contemplan circunstancias adicionales, como el pago de créditos por adquisición de bienes materiales para uso y disfrute en beneficio del menor, lo que puede producir necesidades en el alimentante que incluso pongan en riesgo su propia subsistencia. Se adiciona a esto lo reconocido en la sentencia 67-12-SEP-CC la cual impone límites a la aplicación del principio del interés superior del niño, de manera que pueda ser vencible ante estas circunstancias analizadas en líneas anteriores, lo que en conjunto demuestra la necesidad de revisión de este particular.

Es indispensable también la reforma del artículo 16 del Código Orgánico de la Niñez y Adolescencia, relacionado con la determinación de subsidios y otros beneficios legales, ya que en la actualidad en su segundo inciso obliga el pago de dos pensiones alimenticias adicionales por parte de los alimentantes, a pesar de que no laboren en relación de dependencia, lo cual resulta en una desproporcionalidad evidente para las personas que no adquieren este beneficio y que viven del trabajo diario lo que en consecuencia vulnera sus derechos. 
El Código de la Niñez y Adolescencia, busca que prime el cumplimiento del interés superior del niño, sin embargo, se tiene que tomar en cuenta también el principio de la igualdad y el de proporcionalidad reconocidos a nivel constitucional. En este contexto, lo que determina el Código Orgánico de la Niñez y Adolescencia no guarda armonía con la Constitución, por lo que se genera un vacío legal que en esta propuesta de solución se pretende satisfacer. Al realizar esta reforma no se pretende vulnerar el principio del interés del menor, sino se trata de evitar la vulneración de derechos y principios contenidos en la Constitución, en este caso particular, el de los alimentantes que se encuentren desempleados, que no trabajan en relación de dependencia, y no están en la condición de cumplir con esta obligación en cuanto a las pensiones alimenticias adicionales.

\section{Considerando}

Que el Art. 76 de la Constitución de la República determina que en los procesos que establezcan deberes y derechos de cualquier índole, se garantizará además el debido proceso que consta de garantías esenciales. El numeral 6 de la presente norma determina la proporcionalidad debida que existe entre las sanciones e infracciones penales o de cualquier materia e incluso en el ámbito administrativo.

Que el Art. 8 de la Declaración Universal de Derechos Humanos establece que las personas pueden acceder e interponer recursos ante las judicaturas que tengan la competencia debida, de manera que se garanticen los derechos reconocidos por la carta magna cuando se hayan violado derechos fundamentales por cualquier acto ilegítimo que les cause perjuicio.

Que el Art. 3 de la Ley Orgánica de Garantías Jurisdiccionales y Control Constitucional establece que todas las normas contenidas en la Constitución se deben interpretar en la forma que mejor se ajuste a la Constitución en su integralidad, en efecto, cuando exista duda, se interpretará en un sentido que más beneficie los derechos que reconoce la Constitución y respete la voluntad del pueblo. Dentro de este marco, el segundo inciso de la ley en mención indica como método de interpretación el principio de 
proporcionalidad y; establece que de existir un conflicto surgido entre normas o principios que no se puedan resolver a través de las reglas de antinomias, se aplicará este principio. Que el Art. 44 de la Constitución de la República del Ecuador determina que "(...), se atenderá al principio de su interés superior y sus derechos prevalecerán sobre los de las demás personas.

Que el innumerado 15 Título V del Código de la Niñez y Adolescencia, por otra parte, establece que sea el Consejo Nacional de la Niñez y Adolescencia (CNNA) en su resolución 001-CNNA-2013 que expide la tabla de pensiones alimenticias y señala los parámetros que deben cumplirse para su elaboración, los mismos que están distribuidos de la siguiente manera: las necesidades esenciales primordiales del alimentado, los ingresos ordinarios como extraordinarios del alimentante, gastos propios y de sus dependientes directos, la distribución del consumo familiar, ingresos de los alimentantes y derechohabientes, y por último la inflación.

Que el innumerado 16 inciso 2 Título V del Código de la Niñez y Adolescencia, manifiesta que, el alimentado recibirá el pago de dos pensiones adicionales que se cancelarán en el mes de septiembre y diciembre de cada año en lo que tiene que ver al régimen de la Sierra, así mismo, en los meses de abril y diciembre la Costa y Galápagos deberán cancelar el pago de estos montos en los términos establecidos en esta ley, y si el alimentante no labora en relación de dependencia, esto no le exonera del pago al que se ve obligado.

En ejercicio de sus facultades previstas en el numeral 6 del Artículo 120 de la Constitución de la República la Asamblea Nacional expide la siguiente:

\section{Reformatoria al Código Orgánico de la Niñez y Adolescencia}

Artículo 1.- Refórmese las siguientes disposiciones del Código de la Niñez y Adolescencia:

Sustitúyase el Art. Innumerado 15 Título $V$ del Código Orgánico de la Niñez y Adolescencia por el siguiente: 
"Parámetros para la elaboración de la Tabla de pensiones Alimenticias.- El Consejo de la Niñez y Adolescencia, definirá la Tabla de Pensiones Alimenticias Mínimas en base a los siguientes parámetros: a) Las necesidades básicas por edad del alimentado en los términos de la presente ley; b) Los ingresos y recursos de él o los alimentantes...; Estructura, distribución del gasto familiar e ingresos de los alimentantes y derechohabientes; y d) Inflación".

El juez, considerará para la determinación del ingreso para la aplicación del porcentaje correspondiente además de la deducción previa del aporte del trabajador a la seguridad social, las deudas crediticias e hipotecarias de bienes materiales adquiridas por el alimentante en beneficio del alimentado, previa su justificación.

Artículo 2.- Sustitúyase el Art. Innumerado 16 inciso segundo del Título V del Código Orgánico de la Niñez y Adolescencia por el siguiente:

"Subsidios y otros beneficios legales.- Además de la prestación de alimentos, el alimentado tiene derecho a recibir de su padre y/o madre, los siguientes beneficios adicionales:

Inciso segundo: Dos pensiones alimenticias adicionales que se pagarán en los meses de septiembre y diciembre de cada año para las provincias del régimen educativo de la Sierra y en los meses de abril y diciembre para las provincias del régimen educativo de la Costa y Galápagos. A excepción de los alimentantes que se demuestre que se encuentran desempleados".

Dado y suscrito, en la sede de la Asamblea Nacional ubicada en el Distrito Metropolitano de Quito, provincia de Pichincha, a los -- días del año 2020.

\section{DISCUSIÓN}

Uno de los fines del trabajo de investigación consistió demostrar la existencia de la vulneración del principio de proporcionalidad en el cálculo de la tabla de pensiones alimenticias que en su análisis se consideró los requisitos que debe cumplir la proporcionalidad para que sea eficaz (test de proporcionalidad), de manera que se pueda establecer una prevalencia de derechos entre el alimentante y alimentado en casos 
particulares y que en la práctica se ha convertido este principio en un método de control constitucional de la actuación que tiene el Estado y la exigencia del requisito de razonabilidad que deben gozar los actos y relacionados con la idea de justicia, para asegurar que las decisiones de los jueces se basen en principios que tengan como objetivo el cumplimiento de los fines conforme a la Constitución.

En consecuencia de este objetivo y de acuerdo con la problemática establecida, los resultados de la encuesta realizada a las dos unidades de análisis demostraron la evidente vulneración del principio de proporcionalidad del alimentante. En efecto, al no existir una correcta reglamentación en la normativa interna, conlleva a los jueces a aplicar el principio de proporcionalidad contenido en la Constitución como un mero enunciado y que en la práctica no es del todo eficaz.

Con los antecedentes antes mencionados y en respuesta a la problemática en estudio, se ve la necesidad de adecuar la normativa interna del Código Orgánico de la Niñez y Adolescencia en sus inmumerandos 15 y 16 de manera respectiva, para llenar este vacío legal y permitir una mejor aplicación de este principio Constitucional, y que no se vean afectados los derechos de los alimentantes en este grado de limitación y restricción de los derechos fundamentales, atendiendo a la razonabilidad y proporcionalidad del derecho afectado. En correspondencia, el administrador de justicia pondere entre uno y otro derecho fundamental en conflicto y no se tome como absoluto los principios que le resguardan al alimentado.

\section{FINANCIAMIENTO}

No monetario.

\section{AGRADECIMIENTO}

A las jueces de la unidad de familia y abogados en libre ejercicio de su profesión. 


\section{REFERENCIAS CONSULTADAS}

Almeida, P., Erazo, J., Ormaza, D., \& Narváez, C. (2019). La aplicación de los derechos humanos en el interés superior. [The application of human rights in the best interest]. Iustitia Socialis, 624-644. doi:DOI: http://dx.doi.org/10.35381/racji.v5i8.594

Asamblea General de las Naciones Unidas. (10 de Diciembre de 1948). Declaración Universal de los Derechos Humanos. [Universal Declaration of Human Rights]. La Asamblea General. Paris, Francia. Obtenido de https://n9.cl/o2t5

Asamblea Nacional Constituyente. (20 de Octubre de 2008). Constitución de la República del Ecuador. [Constitution of the Republic of Ecuador]. Derechos de protección. Montecristi, Manabí, Ecuador: Registro Oficial No. 449. Obtenido de https://n9.cl/hd0q

Asamblea Nacional Constituyente. (20 de Octubre de 2008). Constitución de la República del Ecuador. [Constitution of the Republic of Ecuador]. Niños, Niñas $y$ Adolescentes. Montecristi, Ecuador: Registro Oficial 449. Obtenido de https://n9.cl/hd0q

Asamblea Nacional del Ecuador. (21 de Septiembre de 2009). Ley Orgánica de Garantías Jurisdiccionales y Control Constitucional. [Organic Law of Jurisdictional Guarantees and Constitutional Control]. Métodos y Reglas de interpretación Constitucional. Quito, Ecuador: Registro Oficial Suplemeto 52. Obtenido de https://n9.cl/su7v

Auris, G. (enero de 2018). El pago de la décimo tercer y décimo cuarta pensión alimenticia en el caso del alimentante desempleado. [The payment of the thirteenth and fourteenth alimony in the case of the unemployed person] Recuperado de: https://n9.cl/0p8o. Ambato, Ecuador: Repositorio Institucional UNIANDES.

Barnes, J. (1994). Introducción al Principio de proporcionalidad en el Derecho Comparado. [Introduction to the Principle of proportionality in Comparative Law] Recuperado de: https://n9.cl/si3ch. Sevilla: Unicersidad de Sevilla.

Becerra, O. (17 de Marzo de 2019). blog.pucp.edu.pe. Obtenido de https://n9.cl/qyb3

Behar, D. (2008). Metodología de la Investigación. [ Investigation methodology]. México DF: Shalom. Obtenido de https://n9.cl/v7sb 
Benalcazar, M. (Abril de 2018). El pago de las pensiones alimenticias adicionales y el derecho de igualdad,proporcionalidad e interés superior del niño. [Payment of additional alimony and the child's right to equality, proportionality and best interests] Recuperado de: https://n9.cl/ald. Ambato, Ecuador: Repositorio Institucional UNIANDES. Obtenido de http://dspace.uniandes.edu.ec

Cáceres, R. (2016). El principio de constitucionalidad y las pensiones adicionales de alimentos. [The principle of constitutionality and additional maintenance pensions] Recuperado de: https://n9.cl/v5le. Ambato, Ecuador: Repositorio Institucional UNIANDES.

Cárdenas, J. (2014). Noción, Justificación y Críticas al Principio de Proporcionalidad. [Notion, Justification and Criticisms of the Principle of Proportionality] Recuperado de: https://n9.cl/vwaqa. Mexico DF: Instituto de Investigaciones Jurídicas. Recuperado el 19 de 03 de 2013, de http://www.scielo.org.mx

Congreso Nacional. (03 de Junio de 2003). Código de la Niñez y Adolescencia. [Childhood and Adolescence Code]. Subsidios y otros beneficios legales. Quito, Ecuador: Registro Oficial No. 737. Obtenido de https://n9.cl/0jp1

Consejo Nacional de la Niñez y Adolescencia. (15 de Enero de 2013). Resolución. [Resolution] Recuperado de: https://n9.cl/3s50. Ecuador.

Corte Constitucional. (27 de Marzo de 2012). Sentencia. [Judgment] Recuperado de: https://n9.cl/b1m9c. Ecuador.

Corte Constitucional. (4 de Septiembre de 2013). Sentencia. [Judgment] Recuperado de: https://n9.cl/t4ct. Ecuador.

Estudios Constitucionales. (2012). El Principio de Proporcionalidad en la jurisprudencia del Tribunal Constitucional. [The Principle of Proportionality in the jurisprudence of the Constitutional Court]. Scielo. doi:http://dx.doi.org/10.4067/S071852002012000100003

Ferrajoli, L. (2004). Derecho y Razón. [Right and Reason] Recuperado de: https://n9.cl/mpcq. Madrid: Trotta S.A.

Hernández, R., Fernández, C., \& Baptista, M. d. (2014). Metodología de la Investigación. [Investigation methodology]. México DF: Interamericana Editores, S.A. de C.V. Obtenido de https://n9.cl/65F 
Marco Vinicio Cadme-Orellana; Cecilia Ivonne Narváez-Zurita; Juan Carlos Erazo-Álvarez; José Luis Vázquez-Calle

Igartúa, J. (1998). Discrecionalidad técnica, motivación y control jurisdiccional. [Technical discretion, motivation and jurisdictional control]. Madrid: Civitas S.A.

López Idalgo, S. (2017). El Principio de Proporcionalidad como Canon de Constitucionalidad: Una aproximación al caso ecuatoriano. [The Principle of Proportionality as a Canon of Constitutionality: [An Approach to the Ecuadorian Case]. Estudios de Deusto, 185-217. doi:http://dx.doi.org/10.18543/ed-65(1)2017pp185-217

Ministerio de Inclusión Económica y Social. (12 de Febrero de 2020). Tabla de pensiones alimenticias. [Alimony table] Recuperado de: https://n9.cl/spw8. Obtenido de http://www.ecuadorlegalonline.com/

Mogrovejo, A., Erazo, J., Pozo, E., \& Narváez, C. (2019). Aplicación del Principio de proporcionalidad en la Jurisprudencia de la Corte. [Application of the principle of proportionality in the jurisprudence of the Court]. Iustitia Socialis, 90-118. doi:DOI: http://dx.doi.org/10.35381/racji.v5i8.563

Organización de las Naciones Unidas. (20 de Noviembre de 1989). Convención sobre los Derechos del Niño. [ Children's rights convention]. Interés Superior del Niño. Nuevo Siglo. Obtenido de https://n9.cl/pnq7

Organización de las Naciones Unidas. (20 de Noviembre de 1989). Convención sobre los Derechos del Niño. [Children's rights convention]. Nivel de vida. Nuevo Siglo. Obtenido de https://n9.cl/pnq7

Presidencia de la República. (20 de Julio de 2009). Ley Orgánica de la Función Legislativo. [ Organic Law of the Legislative Function] Recuperado de: https://n9.cl/vm6z9. Calificación de los Proyectos de Ley. Quito, Ecuador: Registro Oficial Suplemento 642.

Simón, F. (2013). Interés superior del menor: técnicas de reducción de la discrecionalidad abusiva. [Best interests of the child: techniques to reduce abusive discretion]. Iuris Dictio. doi:DOI: https://doi.org/10.18272/iu.v18i18.786

Tamayo, J. (2011). La Decisión Judicial Tomo 1. [The Judicial Decision Volume 1]. Bogotá: Biblioteca Jurídica DIKE.

Vilela, S. (2014). Pretensión de Reducción de Alimentos:¿Es posible su ejecución anticipada?. [Claim of Food Reduction: Is it possible its early execution?] Recuperado de: https://n9.cl/2ptx. 13. Chiclayo, Perú: Universidad Católica Santo Toribio de Mogrovejo. 
Iustitia Socialis. Revista Arbitrada de Ciencias Jurídicas.

Año V. Vol. V. №2. Edición Especial. 2020-II

Hecho el depósito de Ley: FA2016000064

ISSN: 2542-3371

FUNDACIÓN KOINONIA (F.K). Santa Ana de Coro, Venezuela

Marco Vinicio Cadme-Orellana; Cecilia Ivonne Narváez-Zurita; Juan Carlos Erazo-Álvarez; José Luis Vázquez-Calle

Villabella, C. (2015). Los Métodos en la Investigación Jurídica. [ Methods in Legal Research]. México DF: Promoción y Fomento Editorial. Obtenido de https://n9.cl/iuarn

Zaidán, S., \& Simón, F. (04 de 05 de 2020). Conversatorio: Pensón Alimenticia y Regulación de Visitas. [Discussion: Food Pensions and Visiting Regulation]. Cuenca, Azuay, Ecuador.

(C)2020 por los autores. Este artículo es de acceso abierto y distribuido según los términos y condiciones de la licencia Creative Commons Atribución-NoComercial-Compartirlgual 4.0 Internacional (CC BY-NC-SA 4.0)

(https://creativecommons.org/licenses/by-nc-sa/4.0/). 